\title{
PREDICTION POSSIBILITY IN THE FRACTAL OVERLAP MODEL OF EARTHQUAKES
}

\author{
Srutarshi Pradhan \\ Saha Institute of Nuclear Physics \\ 1/AF Bidhan Nagar, Kolkata 700064, India \\ spradhan@cmp.saha.ernet.in \\ Pinaki Choudhuri \\ Department of Physics \\ Indian Institute of Science, Bangalore 560012, India \\ pinakic@physics.iisc.ernet.in \\ Bikas K. Chakrabarti \\ Saha Institute of Nuclear Physics \\ 1/AF Bidhan Nagar, Kolkata 700064, India \\ bikas@cmp.saha.ernet.in
}

\begin{abstract}
The two-fractal overlap model of earthquake shows that the contact area distribution of two fractal surfaces follows power law decay in many cases and this agrees with the Guttenberg-Richter power law. Here, we attempt to predict the large events (earthquakes) in this model through the overlap time-series analysis. Taking only the Cantor sets, the overlap sizes (contact areas) are noted when one Cantor set moves over the other with uniform velocity. This gives a time series containing different overlap sizes. Our numerical study here shows that the cumulative overlap size grows almost linearly with time and when the overlap sizes are added up to a pre-assigned large event (earthquake) and then reset to 'zero' level, the corresponding cumulative overlap sizes grows upto some discrete (quantised) levels. This observation should help to predict the possibility of 'large events' in this (overlap) time series.
\end{abstract}

Keywords: Earthquake, fractal, Cantor set, overlap time series 


\section{Introduction}

The two-fractal overlap model of earthquake [1] is a very recent modeling attempt. Such models are all based on the observed 'plate tectonics' and the fractal nature of the interface between tectonic plates and earth's solid crust. The statistics of overlaps between two fractals is not studied much yet, though their knowledge is often required in various physical contexts. For example, it has been established recently that since the fractured surfaces have got wellcharacterized self-affine properties [2-4], the distribution of the elastic energies released during the slips between two fractal surfaces (earthquake events) may follow the overlap distribution of two self-similar fractal surfaces [1,5] (see also [6]). Chakrabarti and Stinchcombe [1] had shown analytically that for regular fractal overlap (Cantor sets and carpets) the contact area distribution follows a simple power law decay.

The two fractal overlap magnitude changes in time as one fractal moves over the other. The overlap (magnitude) time series can therefore be studied as a model time series of earthquake avalanche dynamics [7, 8].

Here, we study the time $(t)$ variation of contact area (overlap) $m(t)$ between two well-characterized fractals having the same fractal dimension as one fractal moves over the other with constant velocity. We have chosen only very simple fractals: regular or non-random Cantor sets and random Cantor sets. We analyse the time series data of Cantor set overlaps to find the prediction possibility of large events (occurrence of large overlaps). we show that the time series $m(t)$ obtained by moving one fractal uniformly over the other (with periodic boundary condition) has some features which can be utilized to predict the 'large events'. This is shown utilizing the discrete or a 'quantum' nature of the integrated (cumulative) overlap over time.

\section{The fractal overlap model}

We consider here the overlap sets of two identical fractals, as one slides over the other. We study the overlap distribution $P(m)$ for the overlap set $m$ defined at various (sliding) positions of the two fractals. First, we consider two regular cantor sets (at finite generation $n$ ).

(a)

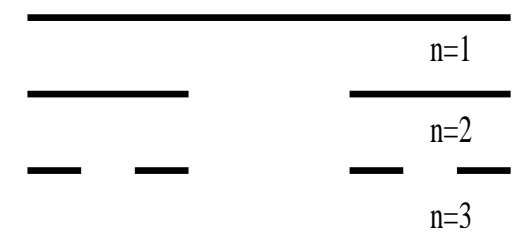

(b)

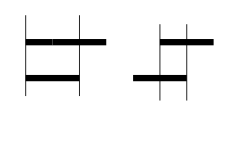


Figure 1. (a) A regular Cantor set of dimension $\ln 2 / \ln 3$; only three finite generations are shown. (b) The overlap of two identical (regular) Cantor sets, at $n=3$, when one slides over other; the overlap sets are indicated within the vertical lines, where periodic boundary condition has been used.

Next, we consider the same for two identical random cantor sets (at finite generations $n$ ).

(a)

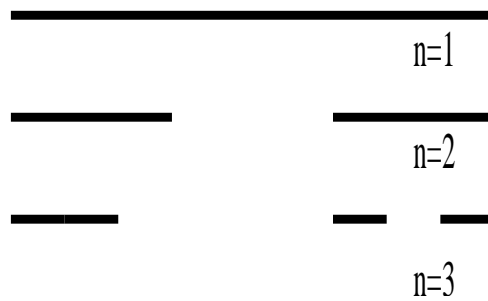

(b)

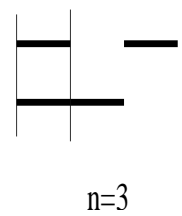

Figure 2. (a) A random Cantor set of dimension $\ln 2 / \ln 3$; only three finite generations are shown. (b) Overlap of two random Cantor sets (at $n=3$; having the same fractal dimension) in two different realisations. The overlap sets are indicated within the vertical bars.

We studied earlier [5] the overlap statistics for regular and random Cantor sets, gaskets and percolating clusters [9]. We found a universal scaling behavior of the overlap or contact area $(m)$ distributions $P(m)$ for all types of fractal set overlaps mentioned: $P^{\prime}\left(m^{\prime}\right)=L^{\alpha} P(m, L) ; m^{\prime}=m L^{-\alpha}$, where $L$ denotes the finite size of the fractal and the exponent $\alpha=2\left(d_{f}-d\right) ; d_{f}$ being the mass dimension of the fractal and $d$ is the embedding dimension. Also the overlap distribution $P(m)$, and hence the scaled distribution $P^{\prime}\left(m^{\prime}\right)$, are seen to decay with $m$ or $m^{\prime}$ following a power law (with exponent value equal to the embedding dimension of the fractals) for both regular and random Cantor sets and gaskets: $P(m) \sim m^{-\beta} ; \beta=d$. However, for the percolating clusters [9], the overlap size distribution takes a Gaussian form.

\section{Time series analysis for two Cantor set overlaps}

We consider now the time series obtained by counting the overlaps $m(t)$ as a function of time as one Cantor set moves over the other (periodic boundary condition is assumed) with uniform velocity.

\section{Overlap time series data}

The time series are shown in Fig. 3., for finite generations of Cantor sets of dimensions $\ln 2 / \ln 3$ and $\ln 4 / \ln 5$ respectively. 

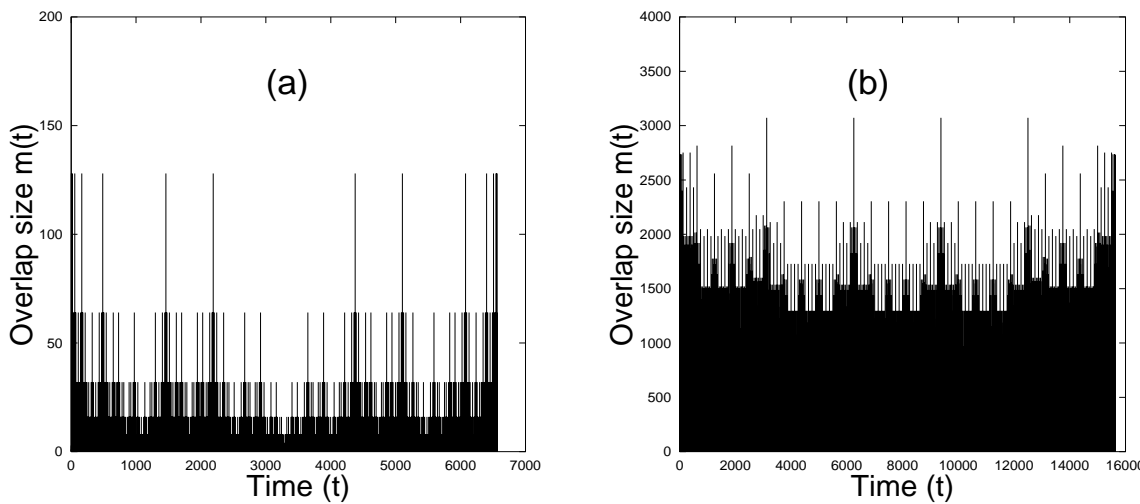

Figure 3. The time $(t)$ series data of overlap size $(m)$ for regular Cantor sets: (a) of dimension $\ln 2 / \ln 3$, at 8 th generation: (b) of dimension $\ln 4 / \ln 5$, at 6 th generation. The obvious periodic repeat of the time series comes from the assumed periodic boundary condition of one of the sets (over which the other one slides).

\section{Cumulative overlap sizes}

Now we calculate the cumulative overlap size $Q(t)=\int_{o}^{t} m d t$ and plot that against time in Fig. 4. Note, that 'on average' $Q(t)$ is seen to grow linearly with time $t$ for regular as well as random Cantor sets. This gives a clue that instead of looking at the individual overlaps $m(t)$ series one may look for the cumulative quantity. In fact, for the regular Cantor set of dimension $\ln 2 / \ln 3$, the overlap $m$ is always $2^{k}$, where $k$ is an integer. However the cumulative $Q(t)=\sum_{i=0}^{t} 2^{k_{i}}$ can not be easily expressed as any simple function of $t$. Still, we observe $Q(t) \simeq c t$, where $c$ is dependent on the fractal. This result is even more prominant in the case of Cantor sets with $d_{f}=\ln 4 / \ln 5$.
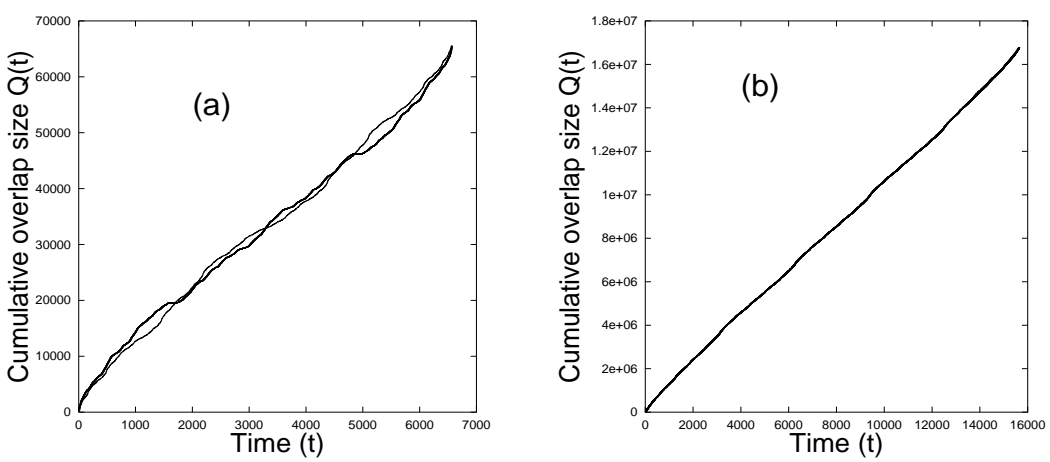

Figure 4. The cumulative overlap $Q$ versus time; for pure cantor sets: (a) of dimension $\ln 2 / \ln 3$ (at 8th generation) and (b) of dimension $\ln 4 / \ln 5$ (at 6 th generation). The dotted line corresponds to those for two identical but random Cantor sets. In (b) the two lines fall on each other. 


\section{Cumulative overlap quantization}

We first identify the 'large events' occurring at time $t_{i}$ in the $m(t)$ series, where $m\left(t_{i}\right) \geq M$, a pre-assigned number. Then, we look for the cumulative overlap size $Q_{i}(t)=\int_{t_{i-1}}^{t} m d t, t \leq t_{i}$, where the successive large events occur at times $t_{i-1}$ and $t_{i}$. The behavior of $Q_{i}$ with time is shown in Fig. 5 for regular cantor sets with $d_{f}=\ln 2 / \ln 3$ at generation $n=8$. Similar results are also given for Cantor sets with $d_{f}=\ln 4 / \ln 5$ at generation $n=6$ in Fig. 6. $Q_{i}(t)$ is seen to grow almost lonearly in time upto $Q_{i}\left(t_{i}\right)$ after which it drops down to zero. It appears that there are discrete (quantum) values of $Q_{i}\left(t_{i}\right)$.
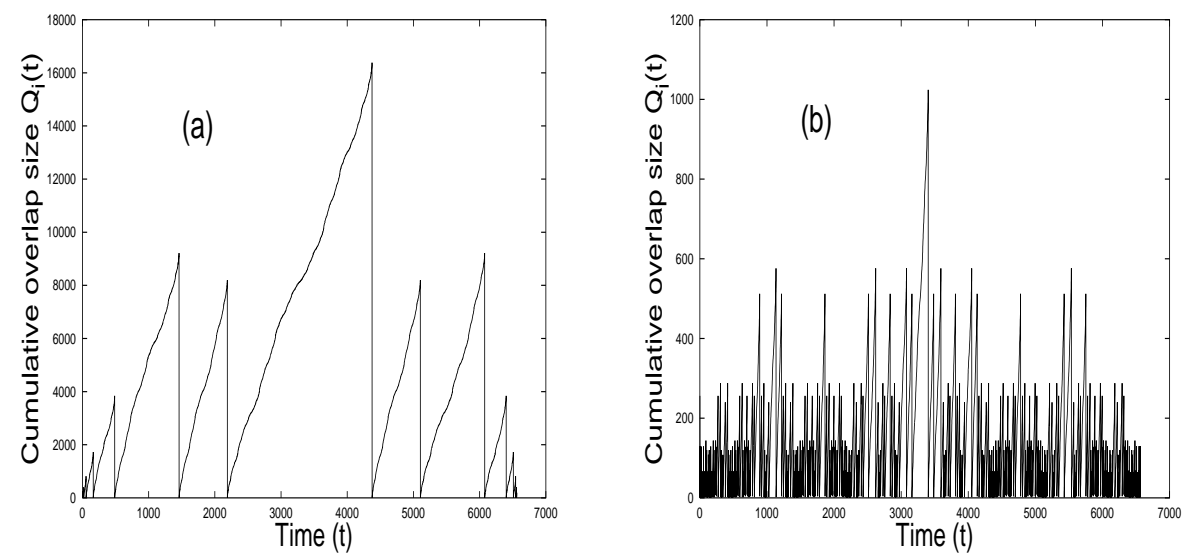

Figure 5. The cumulative overlap size variation with time (for regular Cantor sets of dimension $\ln 2 / \ln 3$, at 8 th generation), where the cumulative overlap has been reset to 0 value after every big event (of overlap size $\geq M$ where $M=128$ (a) and 32 (b) respectively).
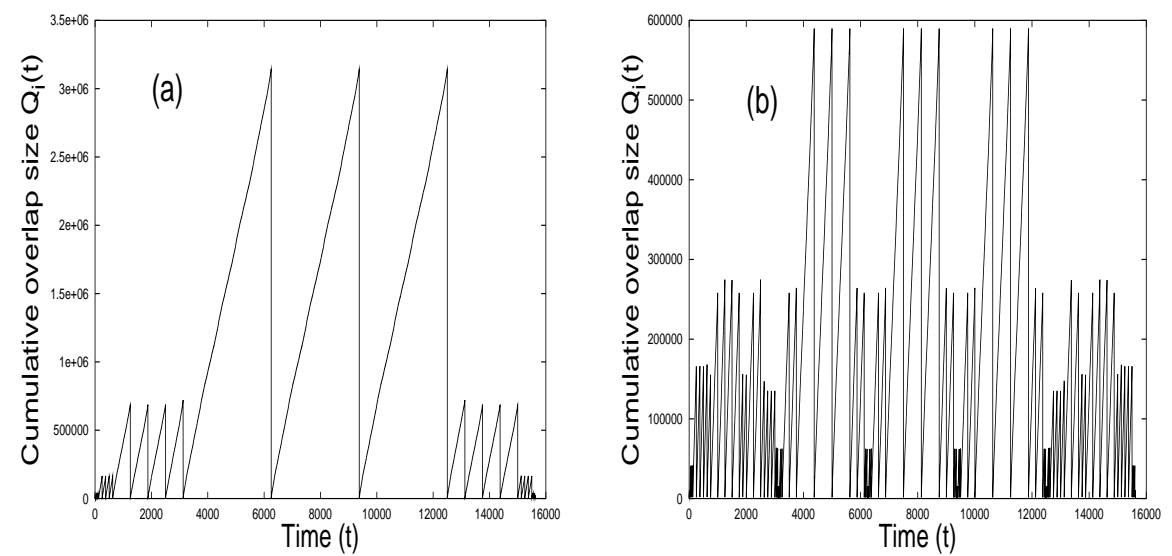

Figure 6. The cumulative overlap size variation with time (for regular Cantor sets of dimension $\ln 4 / \ln 5$, at 6 th generation), where the cumulative overlap has been reset to 0 value after every big event (of overlap size $\geq M$ where $M=2400$ (a) and 2048 (b) respectively). 


\section{Summary and discussion}

If one Cantor set moves uniformly over another, the overlap between the two fractals change quasi-randomly with time (see eg., Fig. 3). The overlap size distribution was argued [1] and shown [5] to follow power law decay. Here we show numerically that if one fixes a magnitude $M$ of the overlap sizes $m$, so that overlaps with $m \geq M$ are called 'events' (or earthquake), then the cumulative overlap $Q_{i}(t)=\int_{t_{i-1}}^{t} m d t, t \leq t_{i}$, (where two successive events of $m \geq M$ occur at times $t_{i-1}$ and $t_{i}$ ) grows linearly with time up to some discrete quanta $Q_{i}\left(t_{i}\right) \cong l Q_{0}$, where $Q_{0}$ is the minimal overlap quantum, dependent on $M$ and $n$ (the generation number). Here $l$ is an integer (see Figs. $5,6)$. Although our results here are for regular fractals of finite generation $n$, the observed discretisation of the overlap cumulant $Q_{i}$ with the time limit set by $n$, is a robust feature and can be seen for larger time series for larger generation number $n$. Similar studies for random Cantor set overlap are in progress. This model study therefore indicates that one can note the growth of the cumulant sesmic response $Q_{i}(t)$, rather than the sesmic event strength $m(t)$, and anticipate some big events as the response reaches the discrete levels $l Q_{0}$, specific to the series of events.

\section{Acknowledgments}

We would like to thank G. Ananthakrishna and P. Bhattacharyya for some useful discussions.

\section{References}

[1] B. K. Chakrabarti, R. B. Stinchcombe, Physica A, 270 (1999)27-34.

[2] B. B. Mandelbrot, The Fractal Geometry of Nature (Freeman, San Francisco, 1982).

[3] B.B. Mandelbrot, D. E. Passoja, A. J. Pullay, Nature 308 (1984) 721-722.

[4] A. Hansen and J. Schmittbuhl, Phys. Rev. Lett. 90, 045504 (2003); J. O. H. Bakke, J. Bjelland, T. Ramstad, T. Stranden, A. Hansen and J. Schmittbuhl, Physica Scripta T106 (2003) 65.

[5] S. Pradhan, B. K. Chakrabarti, P. Ray and M. K. Dey, Physica Scripta T106 (2003) 77.

[6] V. De Rubeis, R. Hallgass, V. Loreto, G. Paladin, L. Pietronero and P. Tosi, Phys. Rev. Lett. 76 (1996) 2599-2602.

[7] R. Burridge, L. Knopoff, Bull. Seis. Soc. Am. 57 (1967) 341-371.

[8] J. M. Carlson, J. S. Langer, Phys. Rev. Lett. 62 (1989) 2632-2635.

[9] See e.g., D. Stauffer and A. Aharony, Introduction to Percolation Theory (Taylor and Francis, London, 1994). 